\title{
ALFRED RUSSEL WALLACE EN EL CENTENARIO DE SU MUERTE
}

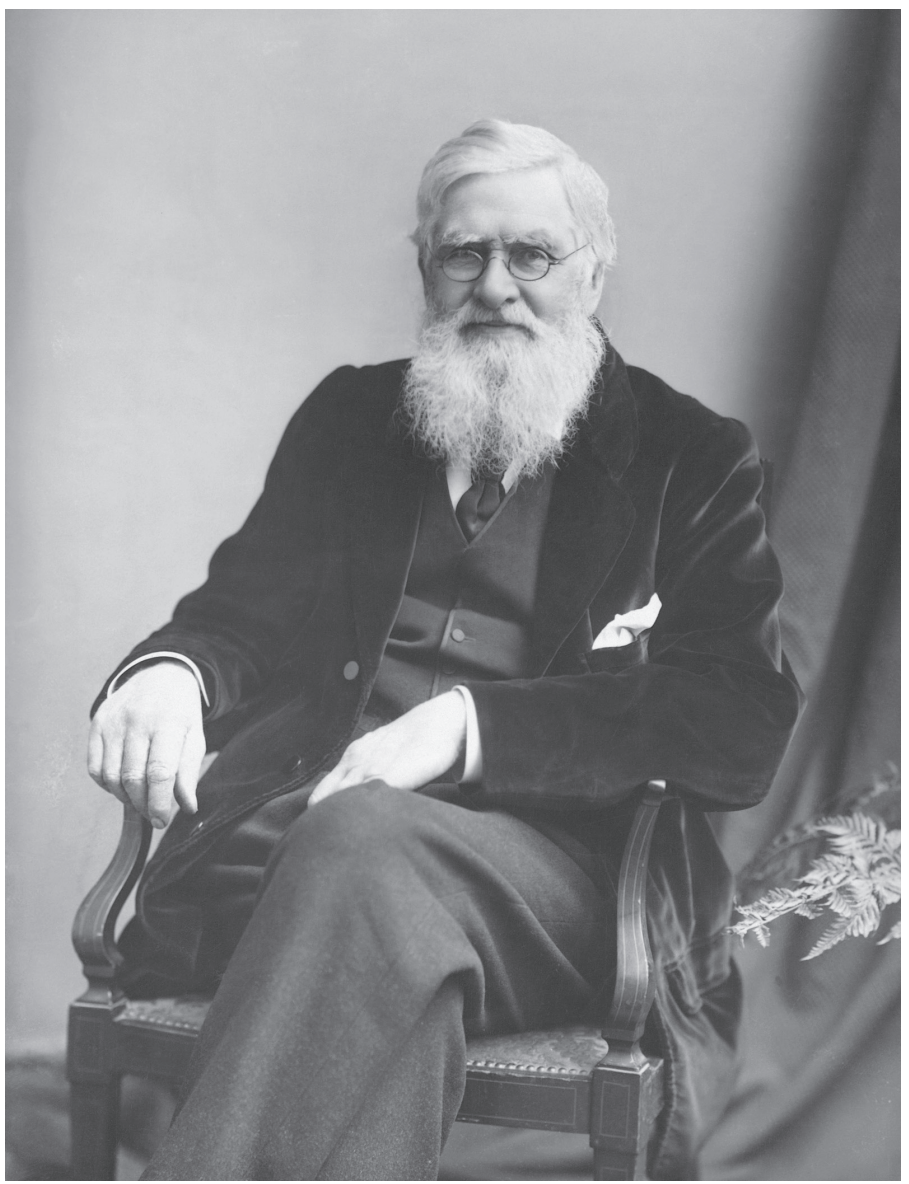

El 7 de noviembre de 1913, Alfred Russel Wallace* muere -durante su sueño- en Broadstone, en las cercanías de Dorset. Inglaterra pierde así uno de sus grandes naturalistas, explorador, biólogo evolucionista, biogeógrafo, antropólogo, y en ocasiones teórico y crítico social, defensor de causas socialistas, polemista, espiritista y opositor a la vacunación y la presencia de vida inteligente en el planeta
Marte que explique los supuestos canales en su superficie.

Noventa años antes, había nacido en Usk, Monmouthshire -ahora Gwent-, en las cercanías de la frontera con Gales, un 8 de enero de 1823. Fue el penúltimo de los nueve hijos, tercero de los cuatro varones, de Thomas Vere Wallace y Mary Anne Greenell. Su padre tenía ascendencia escocesa y su madre inglesa. 
Thomas Wallace fue abogado pero nunca ejerció el derecho dados los ingresos de su propiedad rural, pero ellos resultaron insuficientes cuando su familia creció en número y algunas de sus empresas financieras fueron un fracaso. Por ello la infancia Alfred Russel fue feliz pero con incomodidades económicas que se agravaron con el tiempo hasta obligar su retiro de la educación formal y su incorporación al mercado de trabajo en algunas áreas como la agrimensura, docencia y mecánica bajo la dirección de sus hermanos mayores. Por cierto esa incapacidad del padre por los negocios fue legado a su hijo que tampoco tuvo éxito con los negocios o inversiones.

Gracias a su formación en agrimensura, Wallace descubrió que disfrutaba del trabajo en el campo y se fue interesando en la exploración naturalista del mundo biológico. Pero las lecturas en la biblioteca de la Escuela en que enseñó durante unos años (1843-45) le permitieron familiarizarse con obras como las de Humboldt, Lyell, Chambers, Darwin y Malthus que posteriormente le proveerán de marcos teóricos significativos para sus propuestas evolucionistas.

Su encuentro en 1844 con Henry Walter Bates (1825-1892), entomólogo aficionado con cierto prestigio, le permite formarse como coleccionista y entrever la posibilidad de una carrera de explorador coleccionista. Por ello después de un tiempo le propondrá a Bates un viaje a la Amazonía brasileña para formar colecciones que se venderían en Inglaterra. Por supuesto la lectura de las obras de Humboldt -Personal Narrative- y Darwin -Voyage of the Beagle - son los disparadores de esta motivación- Ambos jóvenes saldrán para Brasil el 25 de abril de 1848, estableciendo su base de operaciones en Pará -actual Belém- y trabajando juntos por dos años, se separan en marzo de 1850, y Wallace se afinca en la región del Río Negro, la que explora con más profundidad que cualquier otro a ese entonces y de la que

* Alfred Russel Wallace. Circa 1895. London Stereoscopic \& Photographic Company (1896).

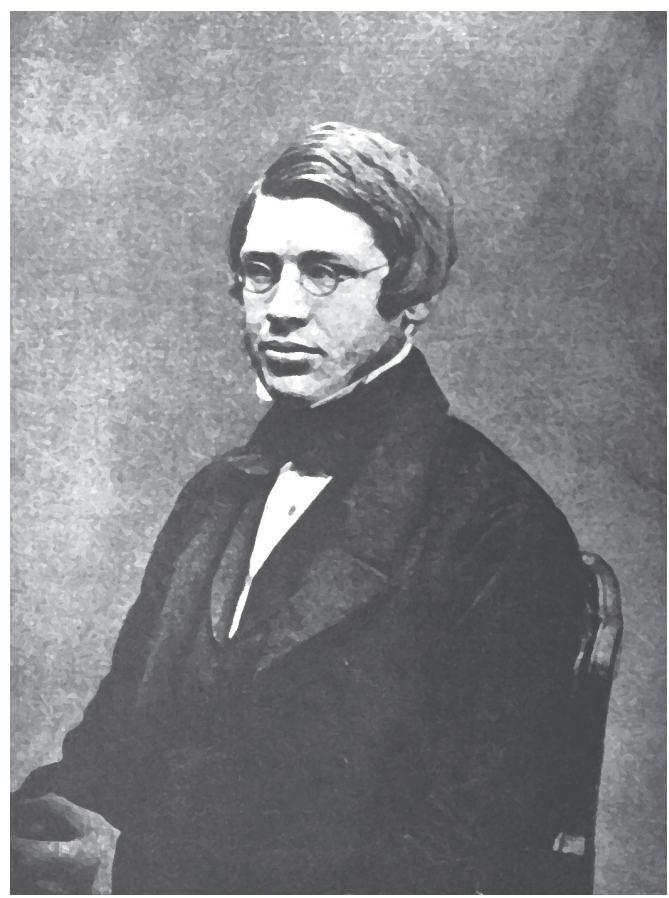

Fig. 2. Wallace a los 24 años de edad, 1848.

hace un mapa que fue referencia básica durante mucho tiempo después. Pero por razones de salud reconoce que no puede explorar más allá y decide regresar a Inglaterra vía Pará, a inicios de 1852. Al llegar a Pará descubre con pesar que su hermano menor, Herbert lo había seguido y había fallecido recientemente. Además que la mayoría de sus colecciones no habían sido enviadas a Inglaterra de manera periódica por lo que debe hacer el embarque de la totalidad. Para julio está en condiciones de embarcarse de regreso pero para su mala fortuna en agosto seis, el barco Helen se incendia y se hunde llevándose al fondo sus colecciones y casi todos los documentos de la expedición -cuadernos de notas y bocetos. El mismo Wallace peligra pues los botes de salvamento son azotados por tormenta y no será sino después de unos diez días que un barco los logra rescatar. El viaje de regreso le toma en condiciones tan difícil unos ochenta días. Llega a Inglaterra el 1 de octubre de 1852. Bates permanece en Brasil por unos siete años más, 1859 , culminando sus estudios 
sobre el mimetismo que le aseguraron un lugar en la historia de la biología.

Wallace pasa el siguiente año y medio en Inglaterra tratando de aprovechar lo poco que rescató de sus colecciones y notas. Cobra un seguro de doscientas libras esterlinas que le permite sobrevivir con lo básico, redacta dos libros que al ser publicados en 1853 por los menos le permiten ingresar en el mundo de los naturalistas viajeros pero que no son un éxito como lo fue el Diario del Beagle de Darwin. Los títulos de esas obras son Palm Trees on the Amazon and Their Uses y A Narrative of Travels on the Amazon River and Rio Negro.

Pronto decide que debe emprender nueva expedición, no solamente para volver a emprender la tarea del coleccionismo científico-empresarial sino muy posiblemente para continuar con sus inquietudes evolucionistas que se habían gestado a partir de la lectura de los Vestigios de la Historia Natural de la Creación, la obra de Robert Chambers referida más arriba, pero que en ese entonces no había sido identificado como su autor. Esta obra hacía un despliegue de argumentos en pro del hecho de la evolución pero también en contra del transformismo Lamarckiano y el inmutabilismo del creacionismo y fue duramente atacada por el establishment científico.

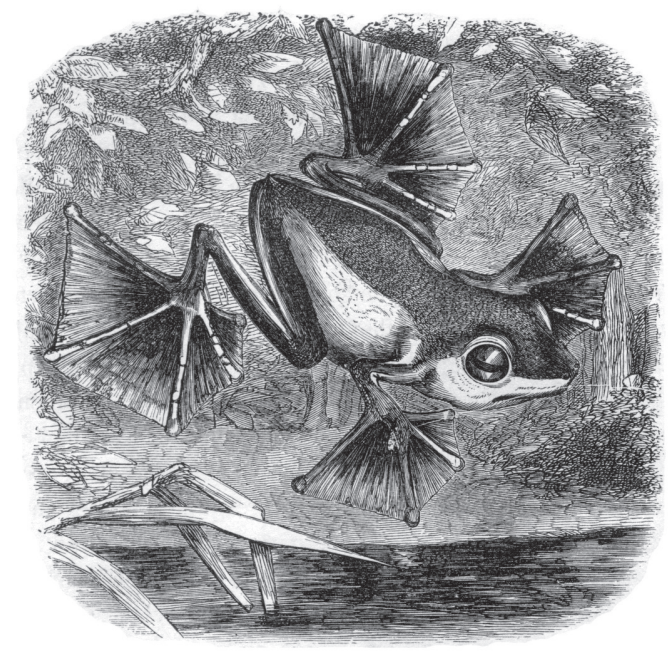

Fig. 3. Rana voladora del libro The Malay Archipelago, de Alfred Russel Wallace.
La nueva meta de Wallace fue Indonesia, o el Archipiélago Malayo, hacia donde se embarca en marzo de 1854, arribando el 20 de abril a Singapore. Luego de ocho años de intenso trabajo de campo y teórico, embarcará de regreso el 20 de febrero del 62, arribando a Inglaterra el 1 de octubre del mismo año. Alrededor de 70 expediciones a diferentes islas y regiones, unas 14.000 millas de recorrido, una colección de 125.660 especímenes con más de mil especies nuevas a incorporar al registro viviente. De toda su experiencia y trabajo publicará innumerables artículos científicos, y finalmente su obra The Malay Archipelago: The Land of the Oang-Utan and the Bird of Paradise en 1869.

Esta obra fundamenta el prestigio de Alfred Russel Wallace como la máxima autoridad sobre este archipiélago por las siguientes décadas, además de llevarlo a muy significativas investigaciones sobre la distribución geográfica de los animales que sirven de base a la zoogeografía posterior. Así, la llamada línea de Wallace, una frontera natural entre las islas con presencia de animales de origen asiático y aquellas con los de origen australiano. Esta separación ahora se comprende mejor a partir de la dinámica de las placas tectónicas. Pero sus estudios pioneros sobre orangutanes y aves del paraíso también son de destacar.

Sin embargo, este período malayo se destaca por sus reflexiones sobre el problema de la transformación de las especies. Estas reflexiones se ejemplifican en dos textos muy significativos de los años de 1855 y 1858 respectivamente, a saber, "On the Law Which Has Regulated the Introduction of New Species" y "On the Tendency of Varieties to Depart Indefinitely From the Original Type".

El primero Wallace lo escribió y envió para su publicación en the Annals and Magazine of Natural History, Volumenr 16, septiembre.

El segundo se escribe y se envía para comentario de Charles Darwin y de Lyell si su valor lo ameritase. No se envía para publicación. Pero se presenta a la Sociedad Linneana el primero de julio de 1858 junto con documentos de Darwin y luego aparecerá publicado en los Proceedings de la Sociedad. 
Esta presentación y publicación se debe a la intervención de Charles Lyell y Robert Hooker luego que son informados por Darwin respecto de la importancia del texto y como implica una teoría equivalente a la propuesta evolucionista que ha construído durante esos últimos "veinte años". Wallace no supo de dicha publicación sino con posterioridad. La excusa que se esgrimió era la lejanía, la tardanza en el correo y la importancia del trabajo. Se puede agregar, por parte nuestra, que la amenaza a la prioridad de Darwin respecto de la formulación de una teoría de la evolución de las especies por selección natural, pudo ser más importante.

El primero de los textos muestra fehacientemente el interés de Wallace sobre la cuestión de la transformación de las especies. Y por ello su publicación atrajo la atención de Lyell, quien se lo hace notar a Darwin dado el conocimiento de las investigaciones de este sobre el mismo tema. Darwin intercabiará cartas con Wallace sobre el texto. Empero, a pesar del título de la publicación, el texto no contiene la ley que explique dicha transformación de especies. En efecto, la ley formulada, a saber, "Cada especie aparecida ha coincidido en el tiempo y en el espacio con otra especie preexistente muy relacionada con ella", no ofrece explicación sino que enfatiza en el hecho de la evolución, dejando por fuera el mecanismo de la misma ${ }^{1}$. Pero ello no es obstáculo para que se pueda afirmar que Alfred Russel Wallace es, para el 1855 , un consumado transformista o evolucionista que busca la solución al "Misterio de los Misterios", que no es simplemente la tesis de la evolución sino la necesidad de una explicación de esta evolución. Pero no ha resuelto todavía dicho problema y por ello es inválido afirmar que la publicación de ese texto implica una instancia pública de la doctrina de la evolución por selección natural. Y no lo es, porque no se hace referencia a la selección natural, sino a patrones geológico-geográficos de divergencia de especies.

El texto de 1858, escrito con gran rapidez mientras sufría de una crisis de salud por fiebres intermitentes, sí contiene una explicación del hecho de la transformación de especies y dicha explicación supone el papel fundamental de la selección natural. En efecto en su autobiografía, My Life, expresa: "En febrero de $1858 \ldots$ el problema (de la evolución) acudió a mi mente, y algo me indujo a pensar en las restricciones positivas descritas por Malthus en su Ensayo sobre la Población, obra que yo había leído hacía unos años y que me había producido una impresión profunda y permanente. Estas restricciones - guerra, enfermedad, hambre, etc.- , pensé, deben actuar tanto sobre los animales como sobre el hombre. Entonces pensé en la multiplicación enormemente rápida de los animales, haciendo que las restricciones resulten mucho más eficaces en ellos que en el caso del hombre, y mientras estaba considerando vagamente este hecho, súbitamente cruzó por mi mente la idea de la supervivencia de los más aptos, la idea de que los individuos eliminados por estas restricciones debían ser en su conjunto inferiores a aquellos que sobrevivían. Bosquejé el borrador de mi artículo... y lo envié por el próximo correo al señor Darwin"2

Ahora bien, dado que este bosquejo de 1858 es la carta de presentación de Alfred Russel Wallace como codescubridor de la teoría de la evolución por selección natural se hace indispensable unos breves comentarios en este momento. Primero, La argumentación de Wallace supone un opositor o defensor de la fijeza de las especies de índole más sofisticada que el tradicional. En efecto, este ya no defiende la inmutabilidad radical de la especie sino que acepta cierta plasticidad de la misma pero bajo el supuesto que dicha plasticidad se da entre ciertos límites y en virtud de ciertas condiciones extremas, pero que superadas esas condiciones, la especie vuelve a su forma original. Como podría ser el caso de una situación artificial de domesticación que lleva al extremo las posibilidades de la especie pero que en su ausencia se vuelve al modelo inicial. Segundo, la propuesta de Wallace se plantea sin referencia esencial a la domesticación, con lo que se evidencia una distinción crucial con el planteamiento darwiniano, para el cual la domesticación es pieza heurística básica. Tercero, igual que en el caso de Darwin, el 
disparador de la solución es la correlación con el pensamiento de Malthus y su énfasis en los factores limitantes del crecimiento de la población y por ende la eliminación de aquellos no aptos para superar tales condiciones hostiles. Se habla de la supervivencia de los más aptos, de los más saludables y fuertes; por supuesto que los débiles, enfermos, muy jóvenes o viejos perecerán fácilmente. Cuarto, se ofrece una explicación de la transformación de una especie cuando resulta que una variedad de la misma presenta una característica o rasgo que hace a sus poseedores más capaces de asegurarse alimentación y por ende reproducción. Dado un intervalo largo, los miembros de esa variedad se impondrán a las variedades o variedad previa no poseedora de dicha ventaja comparativa, con lo que unos aumentarán en número mientras los otros disminuirán y si se mantiene todo igual, finalmente desaparecerán. Quinto, la argumentación se plantea tanto para los miembros de una especie como para las especies dentro de un orden superior o grupo. Sexto, Wallace hace observaciones de tipo poblacional, en un contexto de consideraciones estadísticas, breves pero muy significativas para los desarrollos del evolucionismo en el siglo XX. Séptimo, Wallace establece al cierre de su texto su total oposición al enfoque evolucionista de Lamarck, con lo que su propuesta es mucho más coherente con el principio de la selección natural sin dejar fisuras por las que se pueda posteriormente infiltrar rasgos lamarckianos como sí sucede en el darwinismo. ${ }^{3}$

En conclusión, Wallace se considera justificado al afirmar que "Creemos haber demostrado que hay en la naturaleza una progresión continua de ciertos tipos de variedades apartándose cada vez más de la forma original, y no parece haber motivo para asignar un límite definido a esa progresión, y que el mismo principio que produce este resultado en el estado natural explica también el porqué las variedades domésticas tienden a volver a la forma primaria. Esta progresión, por pasos pequeños, en varias direcciones, pero siempre controlada y balanceada mediante las

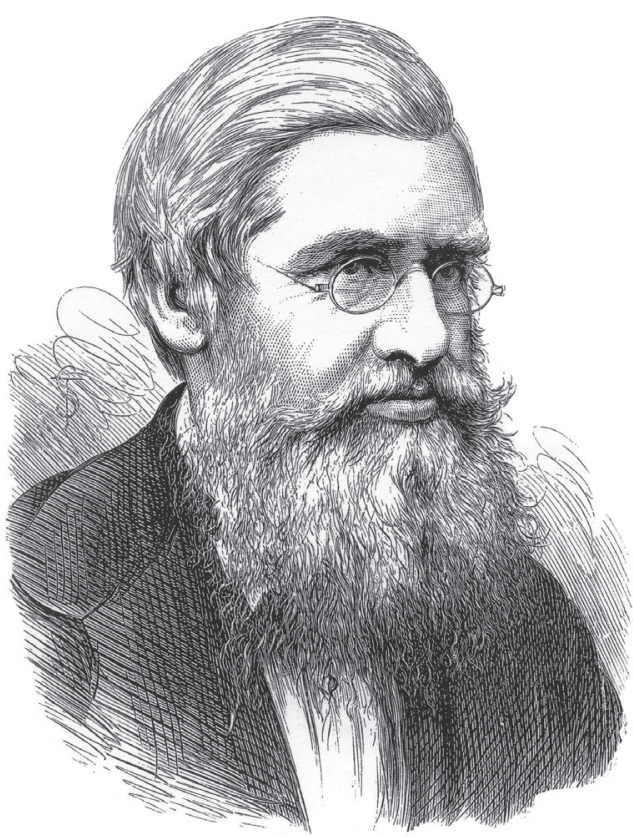

Fig. 4. Wallace en su vejez. Grabado sin datos.

condiciones necesarias, y sólo a la cual está sujeta la supervivencia, puede según se cree, extrapolarse para calzarla a todos los fenómenos presentados por los seres organizados, su extinción y sucesión en las épocas pasadas y todas las extraordinarias modificaciones de forma, instinto y hábitos que exhiben."

Ahora bien, independiente de las razones últimas para la publicación conjunta en la Sociedad Linneana del documento de Wallace y los de Darwin, el hecho es que no causaron gran imprresión. Pero Darwin se enfrentó a la urgente tarea de terminar su obra sobre la evolución de las especies, o al menos, preparar una versión abreviada de la misma. Esa versión más breve y provisional -en su intención inicialserá el Origen de las Especies de 1859. Wallace lo recibirá con gran entusiasmo y a su regreso a Inglaterra en el 62, asumió sin problema que la teoría era de Darwin pues él había tenido un momento de inspiración y probablemente su texto no habría convencido a muchos, mientras el ensayo de Darwin contenía una riqueza de detalles y una línea de argumentación muy sólida. Posteriormente se expresa de la siguiente 
manera: "Por lo que se refiere a la teoría de la selección natural siempre afirmaré que se trata de su teoría y exclusivamente suya. Usted ha elaborado esta teoría con detalles en los yo no hubiera pensado nunca en muchos años. Yo tuve 'un momento lúcido' sobre el tema, y mi trabajo no habría convencido a nadie, a lo sumo solamente hubiera sido considerado como una especulación ingeniosa. Su libro, por el contrario, revoluciona las ciencias naturales y ha arrastrado a los mejores cerebros de nuestra época" 5

Defiende la misma tesis en su libro Darwinism, publicado en 1869 que asegura el nombre de Darwinismo para la doctrina de la evolución por selección natural. El libro se publica después de un largo viaje de Wallace por Estados Unidos y Canadá. En sus conferencias trató temas cruciales del enfoque evolucionista que se convierten en capítulos del libro posterior.

Desde otra perspectiva, el segundo regreso del explorador y naturalista Wallace es muy diferente a su regreso de Brasil. Sus colecciones son enormes, sus notas y documentos una veta interminable para trabajos ulteriores, su incorporación al mundo de los científicos ingleses es plena. Llega a ser parte del grupo reducido pero muy selecto de los amigos de Darwin, en el que junto con Thomas Huxley, Wallace será la fuerza de ataque del darwinismo.

Por supuesto, Wallace también se interesa por asegurar una vida en familia, y se casa con Annie Mitten, hija de un botánico amigo. Procreará tres hijos. El matrimonio fue exitoso pues Annie se interesará en la carrera de su esposo y en muchas ocasiones colaborará con sus labores. En especial en aquellas relativas a la preparación de documentos y en labores de editor que Wallace asumió en algunos tramos de su vida. Y con ello paliaba algunas de las dificultades económicas generadas por su incapacidad de manejarse en el mundo de las finanzas. En fin, que la misma incapacidad de la juventud se mantiene en su largo período de madurez y ancianidad.

Una interminable e importante serie de trabajos y libros aparecerán en los siguientes años, y consolidarán el nombre de Wallace entre los más importantes hombres de ciencia de sus tiempo. Entre los libros se destacan, además del The Malay Archipielago y Darwinism, Contributions to the Theory of Natural Selection (1870), The Geographical Distribution of Animals (1876), Island Life-1880), Man's Place in the Universe (1903), Is Mars Inhabited? (1907) $\mathrm{y}$ The World of Life (1910).

Por supuesto, Wallace no fue nunca un pensador que se conformaba con su pertenencia a cierto modelo o paradigma de ciencia. Por eso muchas veces rompía con los políticamente correcto, como se dice ahora, y conmocionaba su entorno con publicaciones como la de 1874, A Defense of Modern Spiritualism, con la que conmociona a sus colegas, sino que también le sirve de válvula de escape para reforzar una de las grandes diferencias con Darwin, a saber, que la selección natural era el motor de la evolución en el mundo biológico pero no en el humano. Las funciones superiores del hombre, su espiritualidad, no podían explicarse por medio de la selección natural. Para lo humano era necesario trascender ese motor de lo viviente, tanto vegetal como animal, y trascender hacia otros factores explicativos.

También anotemos que Wallace no aceptaba el otro factor de transformación de las especies, la selección sexual.

Pero estas diferencias las consideraba como muy particulares y no como razones para un distanciamiento del pensamiento central del darwinismo, la selección natural como clave para la evolución de lo biológico. Esta adhesión al darwinismo en sentido estricto es plenamente consciente y sincera y ello lo muestra el breve texto que publica en agosto del 1908, "The Present Position of Darwinism". En este ensayo, Wallace responde los ataques en contra del darwinismo por parte del Lamarckismo de nuevo cuño, del mutacionismo de De Vries y de los mendelianos. Estos últimos son caracterizados como defendiendo un núcleo permanente de la especie en virtud de la permanencia de las proporcionalidades de la herencia.

Wallace mantiene su defensa de Darwin en medio del llamado eclipse del darwinismo, que se genera posteriormente a la muerte de 


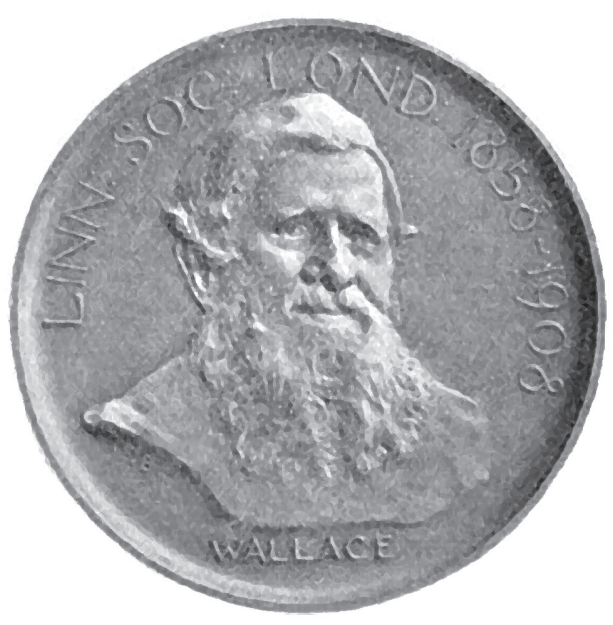

Fig. 5. Medalla Darwin-Wallace, Julio 1908. Es una medalla entregada por la Linnean Society of London en reconocimiento a "los mejores avances en biología evolutiva". Históricamente, esta medalla ha sido entregada cada 50 años, desde 1908.

Darwin, e impulsado por los problemas de la herencia y de la duración temporal de la tierra. En efecto, Fleming Jenkin y Lord Kelvin le plantearon dificultades insalvables para
Darwin. El primero haciendo énfasis en que una explicación del tipo mezcla para la herencia anulaba a largo plazo la importancia de las posibles diferencias individuales, mientras Kelvin mediante cálculos de la capacidad energética del combustible de la Revolución Industrial, el carbón, mostraba que los enormes intervalos de la geología y biología gradualista no eran viables. El sol habría sido grande y tan caliente que habría quemado a la tierra, o por defecto se habría apagado hace tanto tiempo acabando con la vida en el planeta.

A pesar de ello, Wallace no abandona el darwinismo y mantiene su defensa.

Aunque Alfred Russel Wallace hizo patente que no buscaba ni le agradaban honores y más bien buscaba una vida tranquila alejado del bullicio de las grandes ciudades fue objeto de distinciones como doctorados en las universidades de Dublín y Oxford, membresía de sociedades científicas como Fellow de la Royal Society, y recipiente de medallas como la Copley de la Real Sociedad. En la Abadía de Westminster se colocó un medallón con su nombre en el año de 1915 para incorporarlo al panteón de los grandes ingleses.

Guillermo Coronado

Escuela de Filosofía, Universidad de Costa Rica, 2060 San José, Costa Rica, gmocoronado@yahoo.com 


\section{Notas}

1. Una síntesis de este trabajo la presenta Wallace en forma de lista de proposiciones derivadas de la geografía y la geología. "The following propositions in Organic Geography and Geology give the main facts on which the hypothesis is founded.

\section{Geography}

a. Large groups, such as classes and orders, are generally spread over the whole earth, while smaller ones, such as families and genera, are frequently confined to one portion, often to a very limited district.

b. In widely distributed families the genera are of ten limited in range; in widely distributed genera, well marked groups of species are peculiar to each geographical district.

c. When a group is confined to one district, and is rich in species, it is almost invariably the case that the most closely allied species are found in the same locality or in closely adjoining localities, and that therefore the natural sequence of the species by affinity is also geographical.

d. In countries of a similar climate, but separated by a wide sea or lofty mountains, the families, genera and species of the one are often represented by closely allied families, genera and species peculiar to the other.

\section{Geology}

e. The distribution of the organic world in time is very similar to its present distribution in space. f. Most of the larger and some small groups extend through several geological periods.

g. In each period, however, there are peculiar groups, found nowhere else, and extending through one or several formations.

h. Species of one genus, or genera of one family occurring in the same geological time, are more closely allied than those separated in time.

i. As generally in geography no species or genus occurs in two very distant localities without being also found in intermediate places, so in geology the life of a species or genus has not been interrupted. In other words, no group or species has come into existence twice.

j. The following law may be deduced from these facts: -Every species has come into existence coincident both in space and time with a preexisting closely allied species."

2. Mason, Stephen. A History of the Sciences. New York, Collier Books, 1962. En My Life. A Record of Events and Opinions, London, Chapman \& Hall, 1908, la cita corresponde al inicio del capítulo xiii, páginas 189-191.

3. Para un examen mucho más detallado del texto de Wallace, véase mi

"A. R. Wallace, la evolución y su ensayo de 1858". Revista de Filosofia de la Universidad de Costa Rica. Volumen XL, N. ${ }^{\circ}$ 101, Julio-Diciembre, 2002. Páginas 153-162.

4. Traducción de Julián Monge-Nájera en su libro $A B C$ de la evolución, San José, C.R.: EUNED, 1995.

5. Hemleben, Johannes. Darwin. Madrid, Alianza Editorial. 1971. 